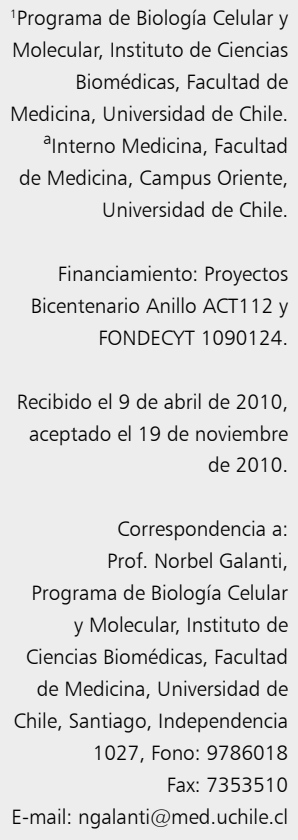

\section{Transmisión de la enfermedad de Chagas por vía oral}

\author{
ALBERTO TOSO M. ${ }^{1, a}$, FELIPE VIAL U., ${ }^{1, a}$, NORBEL GALANTI ${ }^{1}$
}

\section{Oral transmission of Chagas' disease}

The traditional transmission pathways of Chagas' disease are vectorial, transfusional, transplacental and organ transplantation. However, oral transmission is gaining importance. The first evidence of oral transmission was reported in Brazil in 1965. Nowadays the oral route is the transmission mode in 50\% of cases in the Amazon river zone. Oral infection is produced by the ingestion of infected triatomine bugs or their feces, undercooked meat from infested host animals and food contaminated with urine or anal secretion of infected marsupials. Therefore travelers to those zones should be advised about care to be taken with ingested food. In Chile, this new mode of transmission should be considered in public health policies.

(Rev Med Chile 2011; 139: 258-266).

Key words: Chagas' disease; Communicable diseases; Trypanosoma cruzi.
L a enfermedad de Chagas, descrita en 1909 por Carlos Chagas, es causada por el protozoario flagelado Trypanosoma cruzi. Este parásito se transmite principalmente mediante vectores hematófagos triatominos. La enfermedad de Chagas se considera una de las mayores preocupaciones en materia de salud pública en América Latina $^{1}$. Se encuentra en el continente americano desde hace más de 9.000 años, extendiéndose desde el sur de Estados Unido de Norteamérica hasta el sur de Argentina y Chile. Se estima que 15 a 16 millones de personas se encuentran infectadas y que 75 a 90 millones están expuestas a contraer la infección ${ }^{2}$.

En Chile, la enfermedad de Chagas se extiende desde la I a la VI región, incluyendo la Región Metropolitana, siendo más frecuente en áreas rurales y periurbanas ${ }^{3,4}$. En Chile, los principales hematófagos involucrados en la transmisión de Trypanosoma cruzi son artrópodos de la subfamilia Triatominae: el vector doméstico Triatoma infestans y especies silvestres del género Mepraia, conocidos comúnmente con el nombre de vinchuca. Los reservorios en Sudamérica son más de 150 especies de mamíferos, tanto silvestres como domésticos y el hombre ${ }^{5}$.

\section{Formas celulares y ciclo de vida del Trypanosoma cruzi}

Dependiendo del hospedero en que se encuentre, el Trypanosoma cruzi adopta diferentes formas celulares: epimastigote, tripomastigote y amastigote (Figura 1). El epimastigote es la forma extracelular y replicativa presente en el intestino de los triatominos. El tripomastigote es la forma extracelular, no replicativa e infectiva, que se encuentra en la sangre de los mamíferos, en el intestino posterior de los vectores y en sus deyecciones. Los amastigotes son las formas intracelulares replicativas que se encuentran en las células de mamífero ${ }^{3}$. Cuando un triatomino portador del parásito se alimenta de un mamífero, ingiere sangre y simultáneamente defeca. En las deyecciones se encuentran las formas tripomastigotas que ingresan por el lugar de la picadura o por erosiones de la piel y son fagocitados, fundamentalmente por macrófagos. En la célula hospedera, el tripomastigote es incorporado en una vesícula parasitófora, de la que escapa alojándose en el citoplasma, donde se diferencia en amastigote. Esta forma celular inicia numerosos ciclos de división, ocupando el citoplasma de la célula hospedera. Posteriormente, 


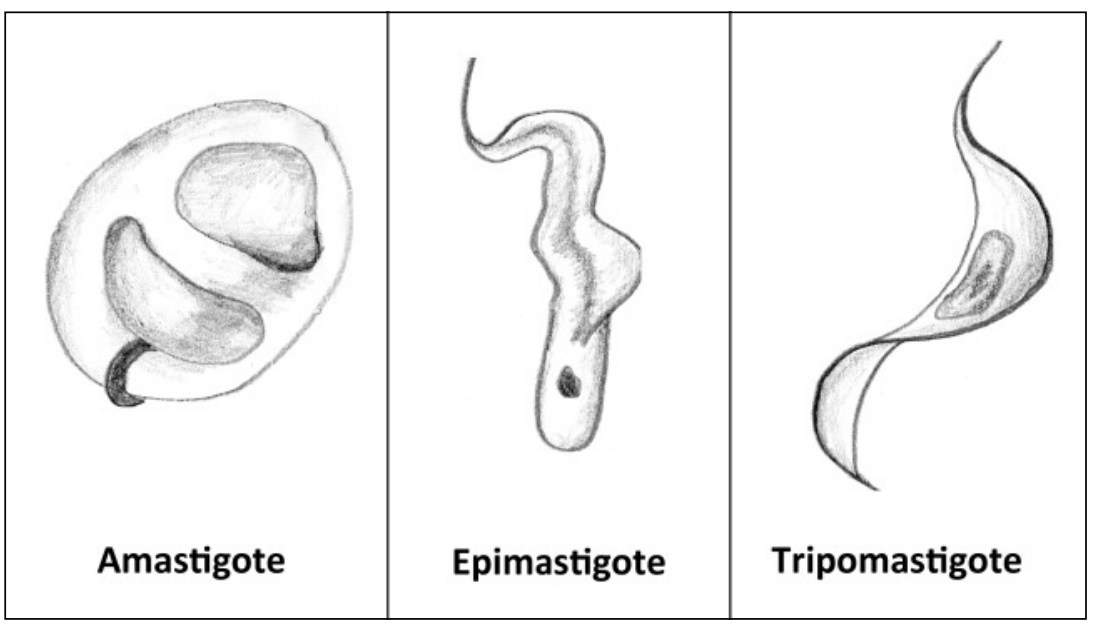

Figura 1. Formas celulares de Trypanosoma cruzi. los amastigotes se diferencian a tripomastigotes altamente móviles, que son liberados al torrente sanguíneo desde donde infectan otras células blanco, tales como ganglionares, musculares y otras $^{6}$. Estos tripomastigotes sanguíneos, cuando son ingeridos por triatominos, se diferencian en el intestino anterior a epimastigotes que se dividen y migran hacia el intestino posterior del insecto. Cuando alcanzan el recto se adhieren a la pared mediante su flagelo y se diferencian en tripomastigotes metacíclicos. Una vez que se despegan de la pared intestinal, son eliminados por las heces de la vinchuca, cerrando el ciclo de vida ${ }^{7}$.

\section{Formas de transmisión y manifestaciones} clínicas

Las formas más tradicionales de contagio de la enfermedad de Chagas son la vectorial, la transfusional, la transplacentaria, los trasplantes de órganos infectados y los accidentes de laboratorio. La transmisión vectorial es causada por las deyecciones de triatominos infectados por Trypanosoma cruzi en la piel de mamíferos, en el momento de alimentarse. Actualmente, con las efectivas medidas que se han tomado para el control de los vectores así como el control de la sangre para transfusiones y de donantes de órganos, en algunos países estas formas de transmisión están controladas ${ }^{8}$. En este contexto, otras formas de contagio han tomado importancia; una de ellas es la transmisión por vía oral debido al consumo de alimentos infectados.

En la enfermedad de Chagas producida por formas tradicionales de infección se distinguen dos fases clínicas; una aguda y una crónica. La fase aguda puede ser asintomática, con síntomas inespecíficos o como un cuadro clínico severo, más frecuente en personas jóvenes. En esta fase, los parásitos se pueden encontrar en sangre y se evidencia por síntomas tales como fiebre, cefalea, anorexia, malestar, mialgia, debilidad, náuseas, vómitos, diarrea, hepatomegalia, esplenomegalia y linfoadenopatía local o generalizada. La fase aguda usualmente es seguida de un período en el que no se presentan síntomas, con una duración variable. Estos pacientes pueden evolucionar a un estado crónico indeterminado, asintomático, o a manifestaciones clínicas tales como cardiopatías, megaformaciones o ambos. Lo más común es que se presenten miocarditis asociadas a arritmias y cardiomiopatías dilatadas. A su vez, pacientes con anormalidades digestivas pueden cursar cuadros de megaesófago o megacolon.

La presentación clínica de la enfermedad de Chagas contraída por transmisión oral es diferente a la observada con las formas tradicionales de infección. Así, después de una latencia de 5 días post-ingesta, se expresa con una manifestación aguda, como resultado de la cual los pacientes desarrollan una miocarditis grave. El cuadro es de alta mortalidad, presentando peor pronóstico mientras menor sea la edad del paciente ${ }^{9}$. 


\section{Epidemiología de la enfermedad de Chagas transmitida por vía oral}

Si bien ya entre los años 1930 y 1950 se consideraba la transmisión por vía oral como un posible mecanismo de transmisión en animales silvestres a través de la ingesta de deyecciones de triatomino ${ }^{10}$, no fue sino muchos años después que se verificó que este mecanismo de transmisión podía involucrar al hombre. Después del trabajo pionero de Schenone y $\mathrm{col}^{11}$ se ha considerado la transmisión de Trypanosoma cruzi vía oral como parte habitual del ciclo enzoótico de este parásito, a través de la ingestión por mamíferos susceptibles de vectores portadores del parásito o de sus deyecciones, así como de reservorios infectados ${ }^{12}$. Actualmente, se cree que esta vía de transmisión es la más importante en la Amazonia de Brasil (50\% de los casos desde 1968 al 2000) ${ }^{13}$.

El primer caso documentado de este tipo de transmisión fue en 1965, en Teutonia, Río Grande del Sur, Brasil, donde se registraron 17 pacientes con enfermedad de Chagas aguda simultánea, sin poder ser explicadas por el mecanismo tradicional de transmisión vectorial. Los análisis anatomopatológicos del músculo cardiaco mostraron la presencia de nidos de Trypanosoma cruzi. Se presume que habrían consumido vegetales contaminados con secreciones de marsupiales infectados ${ }^{10}$.

En 1986, en Catolé do Rocha, Paraíba, se presentaron 26 pacientes con la forma aguda de la enfermedad en forma simultánea, muy similar a lo ocurrido en Teutona. En este caso, tampoco se logró explicar la transmisión por vía vectorial y se concluyó que los pacientes se habrían infectado por beber jugo de caña contaminada con heces de triatomino ${ }^{14-16}$.

A partir de 1996 se asume más expresamente la transmisión de la enfermedad de Chagas por vía oral a partir de un brote de 15 casos autóctonos con enfermedad aguda en el municipio de Mazagâo, Amapá, Brasil ${ }^{10}$. En esa época, ya existía suficiente evidencia sobre la resistencia del parásito a la acidez en el estómago y la posibilidad de transmisión oral en animales, como para considerar este mecanismo posible en humanos ${ }^{10,14,17,18}$.

El 2005 en el estado de Santa Catarina en el sur de Brasil, una zona no endémica, se presentaron 24 casos de enfermedad de Chagas aguda asociados a la ingesta de jugo de caña contaminado con heces de triatominos infectados ${ }^{14,19}$.
Los brotes de Teutonia, Catolé de Rocha y Santa Catarina se dieron en zonas no endémicas de la enfermedad, situación que permitió excluir la presencia del vector y concluir que la forma posible de transmisión era oral. En las zonas endémicas de la Amazonia los brotes son más frecuentes y epidemiologicamente significativos. En un resumen de casos entre 1968 y 2005, en el Instituto Evandro Chagas se evidenciaron 442 casos en 62 brotes distintos asociados principalmente al consumo de jugo de açai. Se presume que este fruto habría sido transportado junto con triatominos y triturados en una máquina para preparar el jugo, contaminándose la bebida ${ }^{14}$.

Durante 2006 se reportaron 94 casos de transmisión de Chagas vía oral en el norte y noreste de Brasil con 6 casos fatales, todos en relación al consumo de jugo de açai o jugo de caña ${ }^{20}$. En la misma región, el año 2007 se reportaron 88 casos con características similares ${ }^{20}$.

En Colombia, hasta el año 2009 se han identificado tres brotes de enfermedad de Chagas aguda, posiblemente asociados a transmisión oral. El primero ocurrió en el poblado de Tibú, Norte de Santander, donde 6 soldados que habían estado previamente en regiones selváticas presentaron miocarditis chagásica, en ausencia de signo de puerta de entrada. Se realizó un estudio a 144 soldados y las posibles situaciones de contagio, pero no se logró determinar con certeza la transmisión por vía oral ${ }^{21}$.

Otro brote se presentó en 1999 en Guamal, Magdalena, Colombia, con 22 casos, de los cuales sólo uno fue verificado anatomopatológicamente mediante muestra de miocardio. El resto de los pacientes presentó síndrome febril prolongado, con y sin síntomas generales. Además, hubo 3 fallecidos con sintomatología similar. Se asoció este brote al consumo de vino de palma contaminado, debido a que en las zonas de extracción de esta bebida se encontraron triatominos infectados con Trypanosoma cruzi21.

Finalmente, la prensa de Bucaramanga, Colombia, reportó en diciembre del año 2008, 9 casos de transmisión de Chagas por vía oral, con dos casos fatales. Un mes después se hizo público el caso, en la misma región, de una niña que se habría contagiado con la enfermedad de Chagas por vía oral, falleciendo posteriormente (datos no publicados).

En Venezuela, el año 2007 se presentó un 
brote en un colegio en la localidad Chichiriviche. Aunque hasta la fecha no hay publicaciones al respecto, el Ministerio de Salud venezolano confirmó el caso. Se trató de 47 estudiantes y 3 profesores contagiados por consumo de jugo de guayaba contaminado, presentando 3 casos fatales ${ }^{22}$. Ese mismo año fueron confirmados 128 casos, uno de ellos fatal, por consumo de jugo de frutas en un colegio en Chacao, Venezuela ${ }^{20}$. El año 2009, las autoridades de salud del estado de Vargas, Venezuela, reportaron 80 casos de enfermedad de Chagas por consumo de jugo de guayaba, con cuatro casos fatales (datos no publicados) ${ }^{23}$.

Si bien la mayoría de los casos de enfermedad de Chagas por transmisión oral se han producido en Brasil, se han detectado casos en otros países como Colombia, Argentina, Venezuela y Ecuador $^{12,14}$. No hemos encontrado registros de transmisión de Chagas por esta vía en Chile (Tabla 1).

A partir de estas evidencias, se han sugerido diversos mecanismos posibles de transmisión por vía oral ${ }^{10,12}$, tales como ingesta de triatominos triturados, de frutas o partes aéreas de vegetales contaminadas con heces de triatominos, de carne o sangre de mamíferos infectados y de secreción anal u orina de marsupiales infectados. También se ha encontrado el parásito en leche humana de pacientes cursando el estadio agudo de la enfermedad de Chagas y hay casos descritos de infección de Trypanosoma cruzi vía oral a través de la leche durante la lactancia materna ${ }^{24,25}$ (Figura 2).

También existe el antecedente de ingesta de carne de animales silvestres infectados que forman parte del reservorio, tales como los marsupiales que han sido cazados y luego ingeridos en forma parcialmente cruda. En Argentina se reportó un caso de un niño de 12 años, que durante una excursión de caza se alimentó exclusivamente de vizcachas y agutíes (animales reservorio); a los 20 días falleció debido a insuficiencia cardiaca como

Tabla 1. Recopilación de casos de transmisión oral de Trypanosoma cruzi

\begin{tabular}{|c|c|c|c|c|c|}
\hline País & Región & n casos & Mecanismo & Año & Referencia \\
\hline \multirow[t]{2}{*}{ Argentina } & & 1 & Carne de animales & & $(26)$ \\
\hline & Chaco & 1 & Sangre quirquincho & & $(10)(14)$ \\
\hline \multirow[t]{9}{*}{ Brasil } & Teutonia, Rio Grande do Sul & 17 & Vegetales & 1965 & $(10)$ \\
\hline & Catolé do Rocha & 26 & Jugo de caña & 1986 & $(14)(15)(16)$ \\
\hline & Santa Catarina & 24 & Jugo de caña & 2005 & $(14)(19)$ \\
\hline & Acre & 3 & Jugo de açai & $1968-2005$ & $(14)$ \\
\hline & Amapá & 61 & Jugo de açai & $1968-2005$ & $(10)(14)$ \\
\hline & Amazonas & 9 & Jugo de açai & $1968-2005$ & (14) \\
\hline & Pará & 217 & Jugo de açai & $1968-2005$ & (14) \\
\hline & Norte - Noreste & 94 & Jugo de açai/caña & 2006 & (20) \\
\hline & Norte - Noreste & 88 & Jugo de açai/caña & 2007 & (20) \\
\hline \multirow[t]{4}{*}{ Colombia } & Tibú, Norte de Santander & 6 & No determinado & & $(21)$ \\
\hline & Guamal, Magdalena & 22 & Vino de palma & 1999 & $(21)$ \\
\hline & Bucaramanga & 9 & No determinado & 2008 & no publicados \\
\hline & Bucaramanga & 1 & No determinado & 2009 & no publicados \\
\hline Ecuador & Secumbios & & Carne de animales & & (14) \\
\hline \multirow[t]{4}{*}{ Venezuela } & Chinchiriviche & 50 & Jugo de guayaba & 2007 & $(22)$ \\
\hline & Chacao & 128 & Jugo de frutas & 2007 & (20) \\
\hline & Vargas & 88 & Jugo de guayaba & 2009 & (23) \\
\hline & Total casos: & 845 & & & \\
\hline
\end{tabular}




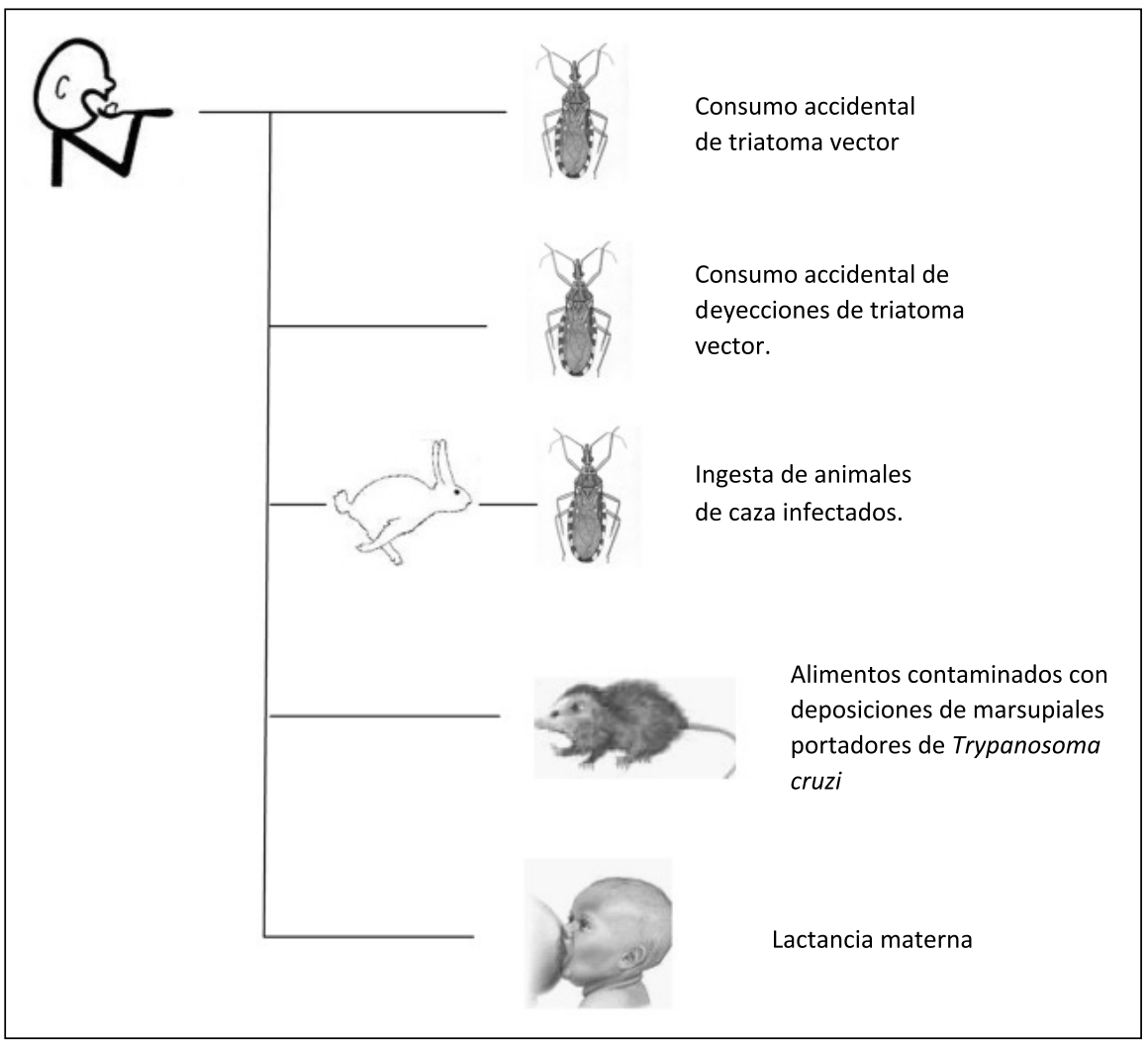

Figura 2. Vías de contagio de la enfermedad de Chagas por vía oral. La ingesta de triatominos ocurre en situaciones en las que éste se mezcla con los alimentos que luego son procesados y rápidamente consumidos pudiendo así, ingresar vía oral. Se ha relacionado con el consumo de vegetales, jugos de fruta y árboles contaminados con deyecciones de triatomi$\operatorname{nos}^{9,10,15,19}$. consecuencia de la intensa miocarditis aguda causada por la infección con Trypanosoma cruzi. La necropsia, además de las lesiones cardiacas características, demostró en el ganglio mesentérico superior una linfadenitis aguda linfomonocitaria e hiperplasia granulomatosa con los caracteres de adenitis aguda chagásica descrita por Mazza en $1940^{26}$. Se estima que el consumo de sangre de animales infectados también podría provocar la transmisión de la enfermedad ${ }^{10}$.

Adicionalmente, los marsupiales infectados presentan tripanosomas en sus glándulas anales, en un estado similar al que se encuentra en los triatominos, con alta densidad de tripomastigotes y epimastigotes. Consecuentemente, se ha observado que la orina y las heces de estos marsupiales tienen la capacidad de transmitir la enfermedad por vía oral por contaminación de alimentos ${ }^{10,12}$ (Tabla 2).

El ingreso del parásito al organismo, según estudios experimentales en ratones, se puede producir a nivel de las mucosas oral, esofágica, gástrica o intestinal ${ }^{12,17,18}$. Así, en el brote de Santa Catarina en 2005, provocado por consumo de jugo de caña contaminado con triatominos infectados, los pacientes afectados presentaban al examen endoscópico lesiones ulceradas con presencia de parásitos a nivel de mucosa intestinal ${ }^{12}$.

Los brotes de enfermedad de Chagas aguda transmitida por vía oral ocurren más frecuente-

Tabla 2. Alimentos asociados a transmisión de la enfermedad de Chagas por vía oral

Jugo de caña (Brasil)

Jugo de açai (Brasil)

Jugo de guayaba (Venezuela)

Vino de palma (Colombia)

Carne de animales de caza (Argentina-Ecuador) 
mente en las épocas más calurosas del año. Esto coincide con una mayor actividad biológica de los triatominos, lo que implica mayor densidad de estos vectores. Consecuentemente, aumenta la contaminación ambiental por heces de triatominos, provocando así un incremento en los casos de enfermedad de Chagas por transmisión oral. Se debe tener en cuenta que los brotes de Catolé do Rocha y Santa Catarina ocurrieron en los meses de octubre y marzo respectivamente. Los brotes de zonas endémicas de Brasil se producen en un $70 \%$ entre agosto y diciembre ${ }^{12}$.

\section{Infectividad del parásito por vía oral. Mecanismo}

Se estima que la máxima capacidad infectiva por vía oral es producida por la forma de tripomastigote metacíclico del parásito, presente en las deposiciones de los triatominos. Esta forma celular sería resistente a la capacidad proteolítica de la mucosa gástrica y tendría la capacidad de adherirse a ella y penetrarla ${ }^{27}$.

Se postula que la capacidad de infectar las mucosas de la vía digestiva deriva de la presencia de una glicoproteína específica del estadío metacíclico del parasito, la gp $82^{27}$. Esta es una molécula de adhesión que se une a las células epiteliales en un mecanismo mediado por receptor e induce movilización de $\mathrm{Ca}^{+2}$, esencial para el ingreso del parásito a la célula ${ }^{28}$. Distintas cepas de Trypanosoma cruzi, en su estadío metacíclico, presentan esta glicoproteína en su superficie ${ }^{29}$.

Similar a gp82 es gp30; sin embargo, esta última, a diferencia de gp82, presenta gran capacidad de infectar células in vitro pero baja afinidad por la mucina gástrica; en consecuencia, es poco efectiva in vivo ${ }^{13,30-32}$ (Figura 3).

Otra glicoproteína parasitaria importante es gp90, que se comporta como modulador negativo de infectividad de células in vitro ${ }^{33}$. Sin embargo, Covarrubias et al. aislaron tres cepas del parásito; una de un paciente infectado por vía oral (SC), otra aislada de marsupiales $(\mathrm{G})$ y una última de Triatoma infestans (CL) y encontraron que la cepa SC presentó mayor expresión de gp90, comparada con las cepas G y CL. Adicionalmente, la cepa SC mostró alta capacidad de infectar ratones vía oral y baja capacidad de infectar células in vitro en comparación a las otras cepas. Finalmente, gp90 de la cepa SC era muy susceptible a la proteólisis por jugos gástricos en comparación con las otras dos cepas, sugiriendo que este sería un factor determinante en la capacidad infectiva por vía oral del parásito ${ }^{33}$.

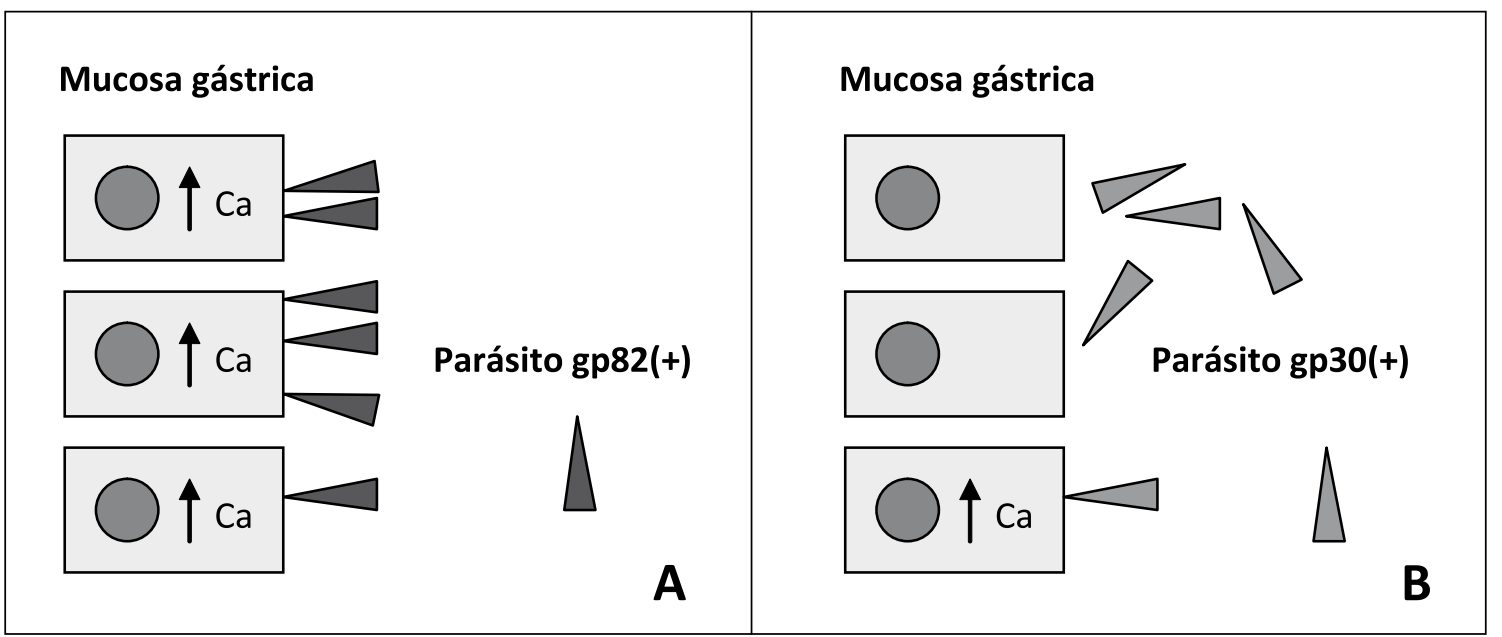

Figura 3. La glicoproteína gp82 específica del estadío metacíclico del parásito permitiría a ciertas cepas de Trypanosoma cruzi atravesar con alta eficiencia la mucosa gástrica en un mecanismo mediado por calcio (A). En contraste, la glicoproteína gp30 presenta una afinidad menor por la mucosa gástrica, disminuyendo la eficiencia de infección de los parásitos por éste mecanismo (B). (Basado en Nobuko Yoshida 2008 "Trypanosoma cruzi infection by oral route. How the interplay between parasite and host components modulates infectivity"13. 


\section{Realidad nacional}

En Chile la situación de la enfermedad de Chagas es diferente a la de otros países de la región; así, como resultado del control de vectores y en los bancos de sangre, la prevalencia ha disminuido verticalmente. En la actualidad, 850.000 personas viven en zonas endémicas de riesgo para contraer la enfermedad y se estima que en el país hay aproximadamente 142.000 personas infectadas con Trypanosoma $\mathrm{cruzi}^{3}$. El 56\% de los casos de Enfermedad de Chagas corresponde al Servicio de Salud de Coquimbo, zona endémica de la enfermedad ${ }^{3}$. La morbilidad ha disminuido desde el año 2000, cuando se observó un ascenso hasta $5,21 \times 100.000$ habitantes. No obstante, la letalidad ha presentado un incremento significativo, de 62,3 x 100.000 habitantes en el año 1990 hasta 290 x 100.000 habitantes en el año $2003^{3}$.

En Chile no se han reportado casos de enfermedad de Chagas producida por transmisión oral. La vigilancia se aplica a los mecanismos de transmisión transfusional y vectorial, mediante el control de la sangre empleada en transfusiones a nivel nacional y de vectores en las zonas endémicas de Chile ${ }^{34}$. Especial atención se presta a la transmisión transplacentaria, por su importancia en la mantención y diseminación de la enfermedad en Chile y fuera de nuestro país ${ }^{35}$. Se han preparado guías clínicas para el diagnóstico y manejo de la enfermedad de Chagas; en estas guías se hace mención a la transmisión por vía oral como mecanismo de contagio pero no se profundiza en el tema a nivel nacional ${ }^{3,4}$.

\section{Prevención}

Considerando que la contaminación de los alimentos se puede producir en su origen, durante su almacenamiento o transporte, así como en su procesamiento, es posible definir ciertas medidas de precaución ${ }^{10}$. Es importante conocer los procesos a que son sometidos los alimentos para aplicar buenas técnicas de prevención. Sin embargo, a pesar de las medidas de control, es muy difícil erradicar por completo este mecanismo de transmisión debido al carácter silvestre de los vectores involucrados. En consecuencia, conocer las diversas características del parásito respecto a su posibilidad de sobrevivir permite organizar de mejor manera las medidas de prevención y control de alimentos.

El Trypanosoma cruzi vive a temperaturas entre $24-28^{\circ} \mathrm{C}$ en los triatominos y entre 36 $37^{\circ} \mathrm{C}$ en mamíferos. Sobrevive 3-24 h en plasma congelado y 1 a 2 semanas en sangre humana a $4^{\circ} \mathrm{C}$. Si se congela a temperaturas bajo $-70^{\circ} \mathrm{C}$ el parásito puede durar años. En leche, jugo de caña u otro líquido a temperatura ambiente es capaz de sobrevivir 20-24 h o más ${ }^{12}$. El pH óptimo oscila entre 7,2 y 7,3; no obstante, el parásito atraviesa la barrera gástrica tolerando bajos $\mathrm{pH}^{12,17}$. Se ha observado también que factores como el etanol, el hipoclorito de sodio y la luz UV, entre otros, eliminan al Trypanosoma cruzi ${ }^{12}$. Sin embargo, posiblemente debido a la presencia de una malla de microtúbulos subyacente a la membrana plasmática $^{36}$, el parásito presenta gran resistencia a la acción de agentes tóxicos.

Especial atención debería focalizarse en animales silvestres de nuestro país, particularmente vizcachas, que pueden estar contaminados con Trypanosoma cruzi. El consumo de carne mal cocida de estos animales podría ser fuente de infección humana.

\section{Conclusiones}

Debido a que la transmisión oral de T. cruzi se investiga solamente en brotes en los que se ha descartado la posibilidad de transmisión por los mecanismos tradicionales, es difícil estimar la magnitud del problema; es probable que exista un subdiagnóstico de la infección por esta vía. El aumento de casos de enfermedad de Chagas transmitida vía oral podría deberse al aumento de factores y situaciones de riesgo, a la mayor capacidad de diagnóstico epidemiológico y gnosológico permitiendo caracterizar casos y brotes o a la mayor visibilidad de la transmisión oral, debido a la disminución de los otros mecanismos de contagio, principalmente el vectorial y el transfusional ${ }^{37}$. No obstante, las autoridades de salud y los especialistas, deberían estar atentos a casos de Chagas con características no clásicas, incorporando en sus protocolos de prevención, diagnóstico y tratamiento la posibilidad de la vía oral de transmisión de esta enfermedad. 


\section{Referencias}

1. Texeira ARL, Nitz N, Guimaro MC, Gomes C, SantosBuch CA. Chagas disease. Postgrad Med J 2006; 82: 788-98.

2. Coura JR. Chagas disease: What is known and what is needed: a background article. Mem Inst Oswaldo Cruz 2007; 102 Suppl 1:113-22.

3. Apt W, Heitmann I, Jercic MI, Jofré L, Muñoz P, Noemí I, et al. Guías clínicas de la Enfermedad de Chagas. Parte I. Introducción y epidemiología. Rev Chil Infect 2008; 25: 189-93.

4. Apt W, Heitmann I, Jercic MI, Jofré L, Muñoz P, Noemí I, et al. Guías clínicas de la Enfermedad de Chagas. Parte II. Enfermedad de Chagas en el adulto, la infancia y adolescencia. Rev Chil Infect 2008; 25: 194-9.

5. Olea A. 1998. Situación epidemiológica de Chagas en Chile. [en línea] <http://epi.minsal.cl/epi/html/public/ chagaschile.htm $>$ [consulta: 29-04-09].

6. Andrade LO, Andrews NW. The Trypanosoma cruzihost-cell interplay: location, invasion, retention. Nat Rev Microbiol 2005; 10: 819-23.

7. Tyler KM, Engman DM. The life cycle of Trypanosoma cruzi revisited. Int J Parasitol 2001; 5-6: 472-81.

8. Cabello C, Cabello F.. Zoonosis con reservorios silvestres: Amenazas a la salud pública y a la economía. Rev Med Chile 2008; 136: 385-93.

9. Barbosa PRB. The oral transmission of Chagas' disease: An acute form of infection responsible for regional outbreaks. Int J Cardiol 2006; 112: 132-3.

10. Silveira A. Factores de riesgo implicados en la transmisión oral de la Enfermedad de Chagas. In: Informe Final Consulta Técnica e Epidemiología, Prevención y Manejo de la Transmisión de la Enfermedad de Chagas como Enfermedad Transmitida por Alimentos (ETA); 4-5 mayo 2006; Rio de Janeiro. Pp. 16-9.

11. Schenone H Jr, González H, Schenone H, Rojas A. Infección experimental de ratas com Trypanosoma cruzi por vía oral. Bol Chile Parasit 1982; 37: 2-9.

12. Dias JC. Notas sobre o Trypanosoma cruzi e suas características bio-ecológicas, como agente de enfermidades transmitidas por alimentos. Rev Soc Bras Méd Trop 2006; 39: 370-5.

13. Yoshida N. Trypanosoma cruzi infection by oral route How the interplay between parasite and host components modulates infectivity. Parasitol Int 2008; 57: 105-9.

14. Valente SA, Valente VC, Pinto A. Epidemiologia e transmissão oral da doença de Chagas na Amazonia Brasileira. Instituto Evandro Chagas. Rodovia. In: Informe Final Consulta Técnica y Epidemiología, Prevención y Manejo de la Transmisión de la Enfermedad de Chagas como Enfermedad Transmitida por Alimentos (ETA); 4-5 mayo 2006; Rio de Janeiro. Pp. 21-6.

15. Shikanai-Yasuda MA, Marcondes CB, Guedes LA, Siqueira GS, Barone AA, Dias JC, et al. Possible oraltransmission of acute Chagas-Disease in Brazil. Rev. Inst. Méd. Trop. Sao Paulo 1991; 33: 351-7.

16. Shikanai-Yasuda MA. Transmissão da doença de Chagas aguda por vía oral: surto epidêmico em Catolé do Rocha, Paraíba, 1986. Hospital das Clínicas da Faculdade de Medicina da Universidade de São Paulo (FMUSP), Depto. Moléstias Infecciosas e Parasitárias da FMUSP.In: Informe Final Consulta Técnica e Epidemiología, Prevención y Manejo de la Transmisión de la Enfermedad de Chagas como Enfermedad Transmitida por Alimentos (ETA); 4-5 mayo 2006; Rio de Janeiro. Pp. 35-8.

17. Hoft DF, Farrar PL, KratzOwens K, Shaffer D. Gastric invasion by Trypanosoma cruzi and induction of protective mucosal immune responses. Infect. Immun. 1996; 64: 3800-10.

18. Hoft DF. Differential mucosal infectivity of different life stages of Trypanosoma cruzi. Am J Trop Med Hyg 1996; 55: 360-4.

19. Steindel M, Kramer Pacheco L, Scholl D, Soares M, de Moraes MH, Eger I, et al. Characterization of Trypanosoma cruzi isolated from humans, vectors, and animal reservoirs following an outbreak of acute human Chagas disease in Santa Catarina State, Brazil. Diagn Microbiol Infect Dis 2008; 60: 25-32.

20. Pereira KS, Schmidt FL, Guaraldo AM, Franco RM, Dias VL, Passos LA. Chagas disease a foodborne illnes. J Food Prot 2009; 72: 441-6.

21. Nicholls R. Enfermedad de Chagas como enfermedad transmitida por alimentos: La experiencia en Colombia. Grupo de Parasitología Instituto Nacional de Salud, Colombia. In: Informe Final Consulta Técnica e Epidemiología, Prevención y Manejo de la Transmisión de la Enfermedad de Chagas como Enfermedad Transmitida por Alimentos (ETA); 4-5 mayo 2006; Rio de Janeiro. Pp. 13-4.

22. International Society for Infectious Diseases. 2009. http://www.promedmail.org. http://www.promedmail. org/pls/otn/f?p=2400:1202:739949417747902::NO: :F2400_P1202_CHECK_DISPLAY,F2400_P1202_PUB_ MAIL_ID:X,76922. [Consultado el 27 de Octubre de 2009].

23. C.A. Editora El Nacional, Venezuela. 2009. http://el-nacional.com/www/site/p_contenido.php?q=nodo/77611/ Gran\%20Caracas/Mal-de-Chagas-agudo-afecta-a-80personas. [Consultado el 13 de Octubre de 2010].

24. Ferreira Cs, Martinho Pc, Amato Neto V, Cruz RRB. Pasteurization of human milk to prevent transmission 
of Chagas disease. Rev. Inst. Med. Trop. S. Paulo 2001;43 (3): 161-162.

25. Mazza S, Montana A, Benitez C, Janzi E. Transmisión del Schizotripanum cruzi al niño por leche de madre con Enfermedad de Chagas. MEPRA 1936; 28: 41-49.

26. Storino R, Jorg ME. Vías de infección y aspectos clínicos. En: Storino R, Milei J (orgs) Enfermedad de Chagas. Buenos Aires, Doyma Argentina; 1994. p. 132-141.

27. Neira I, Silva FA, Cortez M, Yoshida N. Involvement of Trypanosoma cruzi Trypomastigote Surface Molecule gp82 in Adhesion to Gastric Mucin and Invasion of Epithelial Cells. Infect Immun 2003; 71: 557-61.

28. Ruiz R, Favoreto S, Dorta ML, Oshiro ME, Ferreira AT, Manque PM, et al. Infectivity of Trypanosoma cruzi strains is associated with differential expression of surface glycoproteins with differential $\mathrm{Ca}^{2+}$ signaling activity. Biochem 1998; 330: 505-11.

29. Yoshida N. Molecular basis of mammalian cell invasion by Trypanosoma cruzi. An Acad Brás Cienc 2006; 78: 87111.

30. Cordero E, Gentil L, Crisante G, Ramírez Jl, Yoshida N, Añez N, et al. Expression of GP82 and GP90 surface glycoprotein genes of Trypanosoma cruzi during in vivo metacyclogenesis in the insect vector Rhodnius prolixus. Acta Trop 2008; 105: 87-91.

31. Cortez M, Neira I, Ferreira D, Luquetti AO, Rassi A, Atayde V, et al. Infection by Trypanosoma cruzi metacyclic forms deficient in gp82 but expressing a related surface molecule, gp30. Infect Immun 2003; 71: 6184-91.

32. Cortez M, Silva MR, Neira I, Ferreira D, Sasso GR, Luquetti AO, et al. Trypanosoma cruzi surface molecule gp90 down regulates invasion of gastric mucosal epithelium in orally infected mice. Microbes Infect 2006; 8: 36-44.

33. Covarrubias C, Cortez M, Ferreira D, Yoshida N. Interaction with host factors exacerbates Trypanosoma cruzi cell invasion capacity upon oral infection. Int J Parasitol 2007; 37: 1609-16.

34. OPS. Iniciativa de los países del Cono Sur. Primera Reunión de la Comisión Intergubernamental para la Eliminación del Triatoma infestans y la interrupción de la transmisión de la tripanosomiasis americana por transfusión. 1992. Buenos Aires, Argentina. Doc OPS/ PNSP/92.18.

35. García A, Bahamonde MI, Verdugo S, Correa J, Pastene C, Solari A, et al. Infección transplacentaria por Trypanosoma cruzi: Situación en Chile. Rev. Med. Chile 2001; vol.129, n.3, pp. 330-2.

36. Souza W de. Structural organization of Trypanosoma cruzi. Mem. Inst. Oswaldo Cruz 2009; vol.104, suppl.1, pp. $89-100$.

37. OPS/OMS. Resultados de los grupos de trabajo. In: Informe Final Consulta Técnica e Epidemiología, Prevención y Manejo de la Transmisión de la Enfermedad de Chagas como Enfermedad Transmitida por Alimentos (ETA); 4-5 mayo 2006; Rio de Janeiro. Pp. 39-41. 